\title{
Negative contrast effect obtained with downshifts in magnitude but not concentration of solid sucrose reward
}

\author{
MITRI E. SHANAB, JOHN FRANCE, and TED YOUNG \\ California State University, Fresno, California 93740
}

\begin{abstract}
In Experiment I, hungry rats first received 10 pellets of either $4 \%$ or $16 \%$ sucrose, and later received one pellet of the same concentration as before. Control subjects received one pellet of either $16 \%$ or $4 \%$ throughout. A significant negative contrast effect was obtained with both concentrations. In Experiment II, three groups received four pellets of either $4 \%, 8 \%$, or $16 \%$ sucrose concentration before receiving the same number of pellets of $8 \%$ concentration. Neither a positive nor a negative contrast effect was found. The results were discussed in terms of both the frustration and generalization-decrement hypotheses.
\end{abstract}

The literature is repleie with studies that have obtained reliable negative contrast in the runway when food was used as the reinforcer (Black, 1968; Dunham, 1968). A negative contrast effect (NCE) occurs when subjects shifted from a large reward to a smaller reward perform at a significantly lower level than control subjects receiving the smaller reward all the time. There has been however, a consistent failure to obtain similar results when a sucrose solution rather than food was used as the reinforcing agent.

When magnitude of the reinforcer was held constant but a shift from a higher to a lower concentration was made, no NCE was reported (Goodrich, 1962; Goodrich \& Zaretsky, 1962; Homzie \& Ross, 1962; Ison \& Rosen, 1968; Rosen, 1966). Similarly, no NCE was obtained based on a downshift in magnitude rather than sucrose concentration (Rosen \& Ison, 1965; Rosen, 1966). Moreover, no NCE was observed based on a downshift in both magnitude and concentration (Barnes \& Tombaugh, 1973; Flaherty, Riley, \& Spear, 1973; Ison \& Rosen, 1968; Rosen, 1966; Rosen \& Ison, 1965).

This consistent failure to obtain NCE following downshifts in either magnitude or concentration of sucrose solutions, or both, appears to run counter to two major interpretations of contrast. Capaldi (1967) has explained the consistent finding of NCE based on downshifts in magnitudes of food reinforcer in terms of generalization decrements. Subjects receiving a large food reinforcer on each trial would experience a greater generalization decrement when shifted to a smaller reinforcer than control subjects receiving the same small reinforcer, thus accounting for the NCE. On the other hand, Amsel (1967) attributed the NCE to the greater interference resulting from the disruptive frustration responses $\left(R_{f}\right)$ experienced by subjects shifted from

This research was supported in part by Faculty Research Grant NSF-GU-3230. Reprint requests should be addressed to Mitri E. Shanab, Department of Psychology, California State University, Fresno, California, 93740. large to small reward relative to control subjects receiving small reward all the time.

Certain ad hoc assumptions have been suggested to incorporate the lack of NCE within the framework of both frustration and generalization-decrement interpretations (Flaherty et al., 1973; Likely, Little, \& Mackintosh, 1971; Rosen, 1966). For example, it was proposed that if it could be assumed that different sucrose solutions produce little, if any, discriminable aftereffects, then the subjects shifted from a high concentration to a low concentration of sucrose would not suffer a greater generalization decrement than control subjects receiving the lower concentration all the time. The same reasoning can be used to explain the lack of NCE when reductions in magnitude rather than in concentration of sucrose solutions are made. As Flaherty et al. (1973) pointed out, this interpretation does not explain why NCE occurs when the dependent variable is the licking response (Vogel, Mikulka, \& Spear, 1968), or the barpress response (Collier \& Marx, 1959; Weinstein, $1970 \mathrm{a}, \mathrm{b})$ rather than the running response.

On the other hand, if it can be assumed that reductions in sucrose reinforcement, whether such reductions involve a change in concentration of magnitude, produce little or no frustration, then the lack of NCE is not surprising. This interpretation too cannot account for the previous finding of NCE based on liquid sucrose reinforcers.

It is, however, conceivable that the discrepancy in the foregoing findings could be due to the use of different intertrial intervals (ITI). Flaherty et al. (1973) have noted that the ITI values involved in the barpressing of the licking response situation are usually much shorter than those involved in the runway situation. Capaldi (1972), using food reinforcement, showed rather conclusively that the NCE was much stronger and more lasting with massed than spaced training. Capaldi (1972) attributed these results to four sources of generalization 


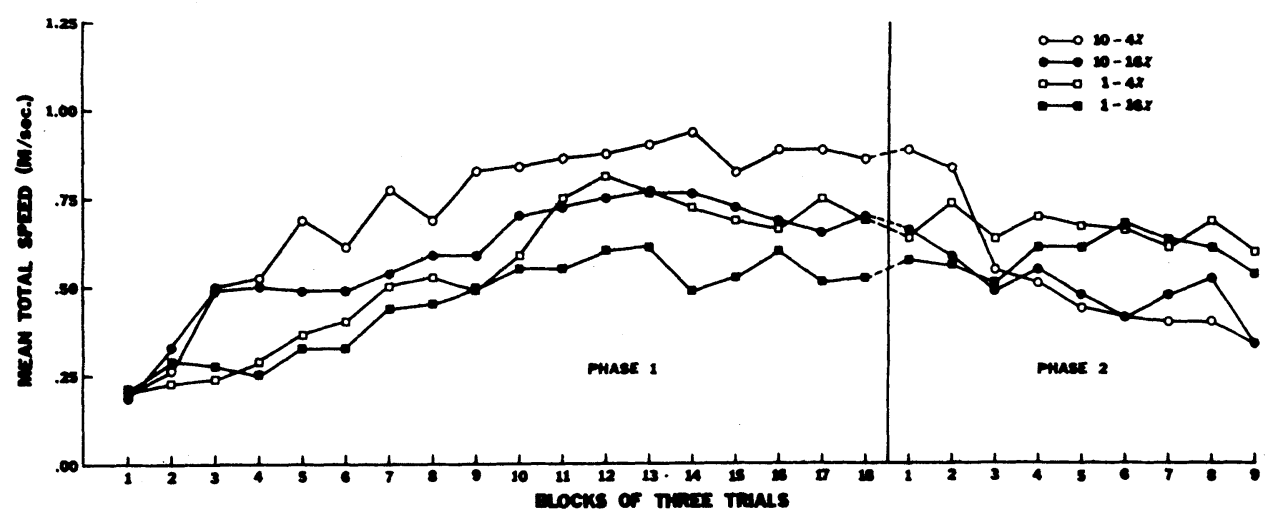

Figure 1. Mean total speed as a function of a downshift in magnitude of solid sucrose reward.

decrement. This new interpretation incorporated the frustration explanation of NCE by assuming that, not only do reinforcer magnitudes have aftereffects which regulate subsequent responding, but so do frustration states which result from reductions or omissions of reinforcement. By further assuming that both reinforcer magnitude and frustration aftereffects are either ITI-dependent (temporary) or ITI-independent (lasting), Capaldi can nicely account for the rather fragile NCE obtained with spaced as compared to massed training. Specifically, Capaldi (1972) stated that, while only two sources of generalization decrement are involved in the spaced-NCE, four such sources are involved in the massed-trial NCE.

The present study was designed to maximize the conditions which produce NCE. Thus, instead of using sucrose in liquid form, sucrose pellets were used and massed training was observed throughout the experiment. Under these conditions, a shift from a large to a smaller number of sucrose pellets should lead to a significant NCE regardless of the concentration used. This outcome is predicted by both Amsel's and Capaldi's theories. A reduction in solid sucrose should produce frustration (Amsel's) or a greater generalization decrement (Capaldi's) in the downshifted relative to nonshifted controls.

\section{EXPERIMENT I}

\section{Method}

Design. Four independent groups of subjects received either 10 or 4 sucrose pellets of either $16 \%$ or $4 \%$ concentration before being shifted to 4 pellets of either $16 \%$ or $4 \%$ concentration.

Subjects. The subjects were 28 naive male albino rats of the Sprague-Dawley strain, approximately 90 days old at the start of the experiment.

Apparatus. A standard Hunter runway was used. The runway was modified in two ways: First, the guillotine doors separating the start from the run and the run from the goal sections were converted from electrical to manual operation. Second, a black self-sticking plastic coating covered the sides and ends of the runway from the outside; the top remained clear Plexiglas.

Four photocells were used to record, start, run, and goal times. The first photocell was located $8 \mathrm{~cm}$ into the runway from the start door. The second photocell was located $25 \mathrm{~cm}$ beyond the first, while the third photocell was placed $100 \mathrm{~cm}$ beyond the second photocell. The distance between the fourth and the third photocell was $25 \mathrm{~cm}$ and was placed $8 \mathrm{~cm}$ into the goalbox. The photocells were connected to electric timers which measured time to the nearest one-hundredth second. The lighting was supplied by two $25-\mathrm{W}$ red light bulbs placed approximately $1.5 \mathrm{~m}$ over the runway. A clear plastic furniture coaster was used as the foodcup. The foodcup was $3.2 \mathrm{~cm}$ in diam and $6.3 \mathrm{~mm}$ deep.

Procedure. Upon arrival from the supplier, the rats were placed on free feeding for 25 days, during which they were weighed and handled each day. Following this time, the subjects were placed on a food deprivation schedule and maintained at about $80 \%$ of their free feeding weight. Water was available at all times in the home cages. After being on deprivation for 2 weeks, each subject was allowed to explore the runway for $2 \mathrm{~min} / \mathrm{day}$. All circuitry was turned off on the first day of exploration. In order to adapt the subjects to the various noises of the apparatus, all subjects received 2 additional days of exploration with all circuitry turned on. Phase 1 was then started. During this acquisition phase, the subjects received three trials per day each according to its group. The subjects were run in squads of three with an average intertrial interval of 3-5 min. The subjects received either 10 pellets of $16 \%$ or $4 \%$ sucrose or 4 pellets of the same concentrations. In Phase 2, the subjects which received a 10-pellet reward of either $16 \%$ or $4 \%$ concentration were shifted to one pellet of the same concentration as before. Each subject received its daily food ration at least $30 \mathrm{~min}$ following its third daily trial.

\section{Results}

Since all time measures were highly similar, all analyses reported here are based on total running speeds derived from reciprocal times.

As Figure 1 shows, the subject apparently reached a stable level before the shift phase was started. This is supported by a two-way analysis of variance with repeated measures over the last four blocks of Phase 1 . While the magnitude factor barely approached significance $[F(1,24)=3.10, .05>p<.10]$, the concentration factor was clearly significant $[F(1,24)=$ $6.02, p<.025]$ the direction of the difference being in favor of the $4 \%$ groups. There was no significant interaction between magnitude and concentration. In 
addition neither the blocks effect $(F<1)$ nor the ensuing interactions with blocks were significant. The absence of a significant block effect indicates that the subjects had reached a stable level by the end of Phase 1 .

Examination of Figure 1 reveals that following a shift from large to small reward, a gradual decrease in performance was observed for both the $4 \%$ and $16 \%$ subjects. It appears that the downshifted subjects did not display the typical NCE until at least 12 trials had elapsed following the shift. However, once this effect was obtained, it persisted until the end of the experiment. The following statistical analyses support these conclusions. During the first three blocks of the shift phase, the concentration factor continued to be significant just as it was before the shift $[F(1,24)=4.26$, $\mathrm{p}<.05]$, but neither the magnitude nor the interaction of magnitude with concentration was significant, both Fs $<1$. However, on Block 4 and thereafter, the concentration factor was no longer significant $(F<1)$. Analyses of variance with repeated measures on the last three, four, and five blocks of trials in Phase 2 yielded almost identical results, so that only the results of the last test will be reported. A highly significant magnitude effect $[F(1,24)=16.78, p<.001]$ was obtained, while neither the concentration nor the Concentration by Magnitude interaction was significant, both $\mathrm{Fs}<1$. The blocks effect did not reach significance $[\mathrm{F}(4,96)=2.42$, $p>.05]$. Nonsignificant results were also obtained for the interaction of Blocks by Concentration $[F(4,96)<1]$, as well as Blocks by Magnitude $[F(4,96)$ $=1.12, \mathrm{p}>.05]$. The three-way interaction was similarly not significant $(\mathrm{F}<1)$. That neither the blocks factor nor the interaction of the blocks with the other factors had any significant effects indicates that all subjects had reached a stable level regardless of their treatment conditions. This in turn suggests that the NCE obtained persisted over the last five blocks of Phase 2 and apparently was not influenced by the particular levels of the independent variables used.

\section{Discussion}

The present study has revealed two interesting findings. In the first phase, it was found that the $4 \%$ groups ran significantly faster than the $16 \%$ groups. This is the reverse of what is usually found when liquid sucrose is used as reward in either the runway or the Skinner box (Flaherty, et al., 1973; Guttman, 1953).

In a recent study which, among other things, compared the rates of barpressing for different concentrations of sucrose solutions relative to regular food reward, Shanab and Gersh (1975), found that hungry rats pressed at a significantly higher rate for food than sucrose reward, regardless of the solutions used $(8 \%, 16 \%$, or $32 \%)$. This finding probably implies that hungry rats prefer food in dry form to sucrose in liquid form. If this is the case, then hungry rats should have a greater preference for $4 \%$ than $16 \%$ sucrose pellet rewards because the former would taste more like "food" than the latter. Hence, the subjects receiving $4 \%$ sucrose pellets should respond at a higher rate than those receiving $16 \%$ pellets, which was the case in this study.

The second major finding is the significant NCE obtained following a reduction in the amount of solid sucrose regardless of the sucrose concentration used $(4 \%$ or $16 \%)$. This result agrees with both the frustration and generalization-decrement interpretations. According to Amsel (1962), the subjects shifted from a large to a small number of sucrose reward pellets should experience an emotional state of frustration which behaviorally would be reflected in avoidance responses that interfere with the learned goal-approach response. In other words, the downshifted subjects would run slower than the nonshifted subjects because of the frustration they experience during the shift. Similarly, Capaldi (1967) proposed that such downshifted subjects would experience a greater generalization decrement than their nonshifted control subjects, which would be reflected in slower running speeds for the former relative to the latter subjects. Thus, both theorists predict a NCE following a reduction in the magnitude of solid sucrose reward.

The present design does not allow for a separate evaluation of the effects of a reduction in either solid sucrose or solid food reward. Because of the rather consistent finding of NCE based on downshifts in the magnitude of solid food reward, the obvious (food only) control groups were not included in the present design. However, while a direct comparison cannot be made between comparable reductions in solid food and sucrose rewards, certain aspects of the present results preclude the tempting conclusion that the obtained NCE was primarily a function of a reduction in magnitude of solid food reward. Downshifts in the magnitude of solid food reward have typically produced abrupt and temporary decrements in performance (Capaldi, 1972). The decrements in performance observed in this study following downshifts in solid sucrose reward were not only gradual but showed no signs of attenuation for a considerable number of trials. It appears, that, like liquid sucrose, solid sucrose also has different behavioral consequences than solid food reward (Bolles, 1967; Tombaugh, McCloskey, \& Tombaugh, 1971).

The fact remains, however, that the NCE obtained in this study could have also been a function of a downshift in solid food reward. It should be noted that besides the sugar content, the main ingredients of the sucrose pellets are the same as those that constitute the regular food pellets, which presents a possible source of confounding. The superiority of the $4 \%$ relative to the $16 \%$ subjects in acquisition might be attributed to the fact that the former subjects receive more food reward.

It is obvious that the animals could discriminate different sucrose concentration in solid form as evidenced by the significant concentration effect in the preshift phase. To test for negative contrast, shifting sucrose concentration would be methodologically a sounder procedure than shifting sucrose magnitude, since the underlying food content would be held constant, or nearly so. Therefore, in the second experiment concentration rather than magnitude of solid sucrose reward was shifted.

\section{EXPERIMENT II}

To control for the possible confounding in the first experiment, independent groups of subjects were trained to receive three different sucrose concentration, then all subjects were given a common sucrose reward. The latter phase provided for tests of both negative and positive contrast based on shifts in concentration of solid sucrose reward. Finally, all subjects underwent extinction.

\section{Method}

Design. Three groups of subjects received either $4 \%, 8 \%$, or $16 \%$ sucrose pellets until stable performance was observed. Then all subjects received $8 \%$ sucrose reward followed by an extinction phase.

Subjects. The subjects were 36 male naive albino rats of the Sprague-Dawley strain, approximately 90 days old at the start of the experiment.

Apparatus. The same apparatus used in Experiment I was used in Experiment II. 
Procedure. The general handling, deprivation, exploration, and other preliminary procedures were identical to those used in Experiment I. The three groups in Phase 1 received four $45-\mathrm{mg}$ Noyes sucrose pellets of either $4 \%, 8 \%$, or $16 \%$ concentration for a total of 36 trials. In Phase 2, all subjects received four 4\% sucrose pellets per trial for a total of 24 trials. All subjects were given 15 extinction trials in Phase 3 . No foodcup was present in the goalbox during extinction. Three trials per day were given throughout all three phases of the experiment. The intertrial interval was approximately 3-5 $\mathrm{min}$. The subject was removed as soon as it consumed its reward during Phases 1 and 2, or after spending $10 \mathrm{sec}$ in the empty goalbox in Phase 3.

\section{Results}

Since all time measures were highly similar, all analyses reported here are based on total speeds. The three groups did not differ in any of the three phases of the experiment. The concentration effect was not significant over the last three blocks of Phase 1 $[\mathrm{F}(2,33)<1]$. An analysis of variance test performed on all eight blocks of Phase 2 similarly yielded a nonsignificant concentration effect $(F<1)$. A one-way analysis of variance test with repeated measures was caried out on all five blocks of Phase 3. Again the concentration effect was not significant $(F<1)$. As would be expected, the blocks effect in extinction was highly significant $[\mathrm{F}(4,132)=70.64, \mathrm{p}<.001]$. However, the Blocks by Concentration interaction effect was not significant $[F(8,132)<1]$, indicating that all three groups extinguished at the same rate.

\section{Discussion}

It is clear that when shifts in concentration of solid sucrose were made in this experiment neither a PCE nor NCE was obtained. This conclusion should be limited to the concentration values as well as the small magnitude of reward used in this experiment. The latter factor probably accounts partly for the lack of a concentration effect in acquisition (Phase 1). The present experiment further showed that the downshift in magnitude of sucrose reward did not have any persistent effects in extinction (cf. DiLollo, 1964).

\section{GENERAL DISCUSSION}

The finding in Experiment I of a NCE based on a shift in magnitude of sucrose reward supports both the frustration and the generalization-decrement hypotheses, in that apparently a downshift in the magnitude of solid reinforcers produce either a state of frustration in the organism or a distinct change in stimulus conditions which lead to a decrement in performance. Apparently when magnitude is held constant, as was the case in Experiment II, mere shifts in concentration of solid sucrose neither produce frustration nor any distinct stimulus aftereffects. This explanation would be consistent with both the frustration and generalization-decrement hypotheses. However, neither hypothesis describes the specific mechanism responsible for the failure of shifts in concentration to produce NCE. It is conceivable that the crucial variable in such shift studies is neither the magnitude nor the concentration variable but merely the consummatory rate variable. But this would not explain the repeated failure in many studies to obtain NCE based on shifts in magnitude of liquid sucrose. The results of Experiments I and II suggest an alternative hypothesis. It seems that, when solid sucrose reward is used, NCE is more likely to be obtained if a shift in magnitude (Experiment I) rather than concentration (Experiment II) is made. The lack of NCE when concentration is shifted suggests that the significant NCE obtained in
Experiment I was probably largely due to the shift in magnitude of solid food rather than solid sucrose. The obvious implication is that hungry rats respond more effectively to the reinforcer that is more appropriate to their state of deprivation.

\section{REFERENCES}

Amsel, A. Frustrative nonreward in partial reinforcement and discrimination learning: Some recent history and a theoretical extension. Psychological Review, 1962, 69, 306-328.

Amsel, A. Partial reinforcement effects on vigor and persistence: Advances in frustration theory derived from a variety of within-subject experiments. In $K$. W. Spence and J. T. Spence (Eds.), The psychology of learning and motivation. Vol 1 , New York: Academic Press, 1967, Pp. 1-65.

Barnes, W., \& Tombaugh, T. N. Another failure to obtain negative contrast following reductions in sucrose reward. Psychological Reports, 1973, 33, 801-802.

Black, R. W. Shifts in magnitude of rewards and contrast effects in instrumental and selective learning: $A$ reinterpretation. Psy chological Review, 1968, 75, 114-126.

Bolles, R. C. Theory of motivation. New York: Harper \& Row, 1967.

Capaldi, E. J. A sequential hypothesis of instrumental learning. In $K$. W. Spence and J. T. Spence (Eds.), The psychology of learning and motivation. Vol. 1, New York: Academic Press, 1967, Pp. 67-156.

Capaldi, E. J. Successive negative contrast effect: Intertrial interval, type of shift, and four sources of generalization decrement. Journal of Experimental Psychology, 1972, 96, $433-438$.

Collier, G., \& Marx, M. H. Changes in performance as a function of shifts in magnitude of reinforcement. Journal of Experimental Psychology, 1959, 57, 305-309.

DiLollo, V. Runway performance in relation to runway and goalbox similarity and changes in incentive amount. Journal of Comparative and Physiological Psychology, 1964, 58, 327-329.

Dunham, P. J. Contrasted conditions of reinforcement: A selective critique. Psychological Bulletin, 1968, 69, 295-315.

Flaherty, C. F. Rily, E. P. \& Spear, N. E. Effects of sucrose concentration and goal units on runway behavior in the rat. Learning and Motivation, 1973, 4, 163-175.

Goodrich, I. P., \& Zaretsky, H. Running speed as a function of concentration of sucrose during pretraining. Psychological Reports, 1962, 11, 463-468.

Goodrich, K. P. Supplementary report: Running speed as a function of sucrose concentration in a prior free-drinking period. Psychological Reports, 1962, 11, 528-530.

Guttman, N. Operant conditioning, extinction, and periodic reinforcement in relation to concentration of sucrose used as reinforcing agent. Journal of Experimental Psychology, 1953. 46, 213-224.

Homzie, N. J., \& Ross, L. E. Runway performance following a reduction in the concentration of a liquid reward. Journal of Comparative and Physiological Psychology, 1962, 55, 1029-1033.

Ison, J. R., \& Rosen, A. J. Extinction and reacquisition performance as a function of sucrose-solution rewards and numbers of acquisition trials. Psychological Reports, 1968 22, 375-379.

Likely, D., Little, L., \& Mackintosh, N. J. Extinction as a function of magnitude and percentage of food and sucrose rewards. Canadian Journal of Psychology, 1971, 88, 128-132.

Rosen, A. J. Incentive shift performance as a function of magnitude and number of sucrose rewards. Journal of Comparative and Physiological Psychology, 1966, 62 , 487-490.

Rosen, A. J., \& Ison, J. R. Runway performance following changes in sucrose rewards. Psychonomic Science, 1965, 2, 335-336.

Shanab, M. E., \& Gersh, W. D. Behavioral contrast as a function of two different reinforcers. Journal of General Psychology, 1975 , in press.

Tombaugh, J., McCloskey, J. L., \& Tombaugh, T. N. Delayed partial reinforcement effect. Canadian Journal of Psychology, $1971,25,419-426$.

Vogel, J. R., Mikulka, R. J., \& Spear, N. E. Effects of shifts in sucrose and saccharine concentrations on licking behavior in the rat. Journal of Comparative and Physiological Psychology $1968,66,661-666$.

Weinstein, L. Negative incentive contrast effects with saccharine vs. sucrose and partial reinforcement. Psychonomic Science, 1970a, 21, 276-278.

Weinstein, L. Negative incentive contrast with sucrose. Psychonomic Science, 1970b, 19, 13-14.

(Received for publication January 13, 1975.) 